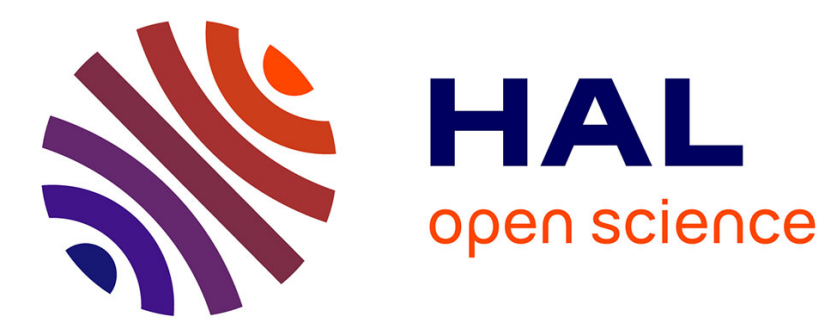

\title{
The Typical Constructible Object
}

Mathieu Hoyrup

\section{To cite this version:}

Mathieu Hoyrup. The Typical Constructible Object. Computability In Europe, Jun 2016, Paris, France. pp.115 - 123, 10.1007/978-3-319-40189-8_12 . hal-01396167

\section{HAL Id: hal-01396167 \\ https://hal.inria.fr/hal-01396167}

Submitted on 14 Nov 2016

HAL is a multi-disciplinary open access archive for the deposit and dissemination of scientific research documents, whether they are published or not. The documents may come from teaching and research institutions in France or abroad, or from public or private research centers.
L'archive ouverte pluridisciplinaire HAL, est destinée au dépôt et à la diffusion de documents scientifiques de niveau recherche, publiés ou non, émanant des établissements d'enseignement et de recherche français ou étrangers, des laboratoires publics ou privés. 


\title{
The typical constructible object
}

\author{
Mathieu Hoyrup \\ LORIA, Inria Nancy Grand Est
}

\section{Introduction}

Baire Category is an important concept in mathematical analysis. It gives a notion of large set, hence a way of identifying the properties of typical objects. One of the most important applications of Baire Category is to provide a way of proving the existence of objects with specified properties without having to give an explicit construction, showing at the same time that these properties are prevalent. For instance it has been extensively used in mathematical analysis to better understand and separate classes of real functions such as analytic and smooth functions (see [9] for a wide range of applications of the Baire Category Theorem in analysis).

This note is about Baire Category in constructive or computable analysis. This subject has been studied in many different directions, for instance in reverse mathematics [3], constructive mathematics [2] or computable analysis [1]. In these fields, one is often interested in studying the properties of "constructible" objects, to separate classes of constructible objects, and to identify the effectiveness of mathematical proofs.

Here we are interested in a particular question: how to apply Baire Category inside classes of constructible objects? Such classes are very small in the sense of Baire Category, as they are countable, so strictly speaking Baire Category cannot be applied inside them. However one can adapt Baire Category and save part of it in these small worlds, and this note illustrates this. We will focus on 4 particular classes of constructible objects, depicted in Figure 1:

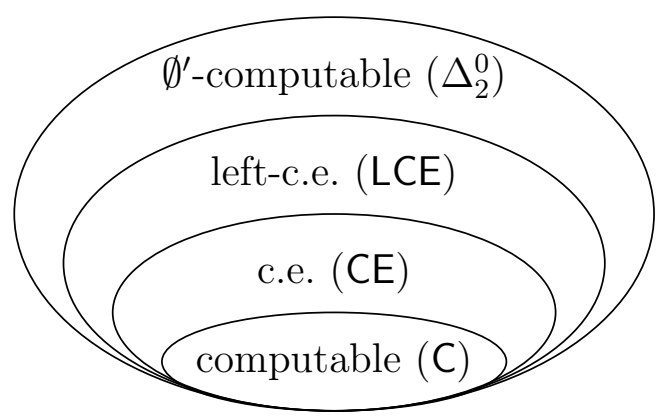

Fig. 1. Four classes of constructible subsets of $\mathbb{N}$ 
- The class $C$ of computable subsets of $\mathbb{N}$.

- The class CE of c.e. subsets of $\mathbb{N}$.

- The class LCE of left-c.e. real numbers in $[0,1]$.

- The class $\Delta_{2}^{0}$ of subsets of $\mathbb{N}$ that are computable relative to the halting problem.

The goal is, for each class of constructible objects, to find an analog of Baire Category to define a notion of large set, of typical or generic object. Why is it interesting? It helps understanding which parts of classical mathematics are still available in a constructive setting. From a more practical perspective, it gives a way to prove the existence of constructible objects with specified properties, avoiding as in the classical setting explicit constructions by using a simpler argument.

For each class $\mathscr{C}$ of objects, we want to investigate the following question:

What does the typical object of $\mathscr{C}$ look like?

The way to do this is to define a notion of small subclass of $\mathscr{C}$. Such a notion should satisfy the following conditions:

Axiom 1. Every singleton $\{A\}$ with $A \in \mathscr{C}$ is small in $\mathscr{C}$,

Axiom 2. The class $\mathscr{C}$ is not small in itself,

Axiom 3. Effective countable unions of small sets are small, for some notion of effectiveness,

Axiom 4. In the hierarchy depicted in Figure 1, a subclass is small in the superclass. In other words, a typical object of the superclass does not belong to the subclass.

A measure-theoretical approach to this problem has been developed by many authors using resource-bounded measure and dimension theory, in particular resource-bounded martingales (see $[11,12]$ for instance). These methods were mainly applied to complexity classes, but not to classes of enumerable objects such as c.e. sets or left-c.e. reals: whether such a development can be done is an interesting problem.

Here we adopt the topological approach of Baire Category. For each class it is done by defining the analog of a nowhere dense subclass by means of effectivization. The meager subclasses are then generated by the nowhere dense classes: they are the subsets of effective countable unions of nowhere dense classes. More precisely in an effective version,

1. Nowhere dense sets are effective, i.e., describable in some way by programs,

2. Countable unions $\bigcup_{i} A_{i}$ are effective, i.e., there is a single program that given $i$ as input, describes $A_{i}$ in the way specified in 1 .

Hence to obtain a notion of meager subclass one simply has to define what is an effective nowhere dense set. Axiom 3. will be automatically satisfied. 


\subsection{Notations and background.}

The set of finite binary strings is denoted by $2^{*}$. The Cantor space $2^{\mathbb{N}}$ is the space of infinite binary sequences, also identified to subsets of $\mathbb{N}$ or reals numbers in $[0,1]$. If $A$ is an infinite binary sequence then $A \uparrow_{n}$ is the prefix of $A$ of length $n$. For each finite binary string $u \in 2^{*}$, the cylinder $[u] \subseteq 2^{\mathbb{N}}$ is the class of infinite binary extensions of $u$. The Cantor space is endowed with the topology generated by the cylinders: the open classes $\mathcal{U} \subseteq 2^{\mathbb{N}}$ are the unions of cylinders. A subclass of $2^{\mathbb{N}}$ is nowhere dense if it is disjoint from a dense open class. A subclass is meager if it is a union of nowhere dense classes.

\section{Computable objects}

Let $C$ be the class of computable subsets of $\mathbb{N}$.

An ordinary nowhere dense set is a set that is disjoint from a dense open set. A natural effective version is then to require the dense open set to be effective, i.e., expressible as a union $\bigcup_{u \in A}[u]$ where $A \subseteq 2^{*}$ is a computably enumerable set.

We then say that a class is meager in $\mathrm{C}$ if it is contained in an effective union of complements of dense open sets. A class is co-meager in $\mathrm{C}$ if it contains an effective intersection of dense open sets.

This notion of nowhere dense set makes the Baire Category theorem computable:

Theorem 2.1 (Baire Category theorem in C). Every class that is co-meager in $\mathrm{C}$ is dense in $\mathrm{C}$, i.e., contains computable elements in every cylinder.

In other words, the class $C$ is not meager in itself and Axiom 2. is satisfied.

Observe that Axiom 1. is also satisfied, i.e., a singleton is meager in C: if $A \subseteq$ $\mathbb{N}$ is computable then $2^{\mathbb{N}} \backslash\{A\}$ is a dense effective open class. Every subclass that can be effectively listed is also meager in $\mathrm{C}$, for instance the class $\mathrm{P}$ of polynomial-time decidable problems.

Example. Consider a map $T$ from $[0,1]$ to $[0,1]$ that is computable. Think of $T$ as a dynamical system: if $x \in[0,1]$ is the state of the system at time $t$ then $T(x)$ is the state at time $t+1$. An initial state $x_{0}$ induces a trajectory defined by $x_{t+1}=$ $T\left(x_{t}\right)$. What do typical trajectories look like?

Now imagine that one simulates $T$ on a computer, computing the trajectory starting from some state $x_{0}$. Of course the computer can only manipulate computable real numbers, so only the computable part of the dynamical system can be observed on the computer, namely the map $T:[0,1] \cap \mathrm{C} \rightarrow[0,1] \cap \mathrm{C}$ (here $\mathrm{C}$ is the set of computable real numbers). What do typical trajectories of the restricted system look like? Are they representative of the original system over $[0,1]$ ?

If the system has a dense trajectory then one can show that all the typical trajectories are dense (i.e., the set of initial states inducing a dense trajectory is 
co-meager), and the computable Baire Category theorem directly implies that the typical trajectories of the restricted system are also dense, in particular there exists a computable dense trajectory.

\section{Computable relative to the halting problem}

Let $\Delta_{2}^{0}$ be the class of subsets of $\mathbb{N}$ that are computable relative to the halting problem.

One can relativize the Computable Baire Category theorem to any oracle, in particular to the halting problem, in a straightforward way. It gives for free a notion of nowhere dense class in $\Delta_{2}^{0}$ : it is a class that is disjoint from a dense open class that is effective relative to the halting problem. Effective unions can be equivalently taken relative to the halting problem or not (the two notions are equivalent by the relativized $s-m-n$ theorem).

Again, Axioms 1. and 2. are satisfied, i.e., the class $\Delta_{2}^{0}$ is not meager in itself and every singleton is meager in $\Delta_{2}^{0}$. Moreover Axiom 4. is also satisfied as the subclass LCE is meager in $\Delta_{2}^{0}$ : the halting problem can effectively list LCE, which is then an effective union of singletons, hence is meager in $\Delta_{2}^{0}$.

We now present a particular class that is co-meager in $\Delta_{2}^{0}$ that has received a lot of attention.

\subsection{1-generic sets}

In Section 2 we mentioned that an ordinary nowhere dense class is a class that is disjoint from a dense open set, which naturally induces the first effective version. A nowhere dense class can equivalently be defined as a subset of the boundary of an arbitrary (i.e., not necessarily dense) open set, which gives another possible effective version: a subset of the boundary of an effective open set.

It gives a strictly weaker notion of nowhere dense class: in general the boundary of an effective open class is not disjoint from a dense effective open class. However, it is always disjoint from a dense open class that is effective relative to the halting problem. As a result, it is meager in $\Delta_{2}^{0}$. As the effective open classes can be effectively enumerated, taking the union of their boundaries gives a class that is meager in $\Delta_{2}^{0}$. Its complement is known as the class of 1-generic subsets of $\mathbb{N}$, and is co-meager in $\Delta_{2}^{0}$. In particular it is non-empty and even dense. It was introduced by Jockush [8] in order to simplify constructions in recursion theory, in the same way as Baire Category simplifies proofs of existence results in mathematical analysis.

While the class of 1-generic sets is just one particular class that is comeager $\Delta_{2}^{0}$, it happens that it captures many interesting co-meager classes in $\Delta_{2}^{0}$, in the sense that it contains them. In other words, many typical properties are already satisfied by the 1-generic sets. Indeed being 1-generic is a kind of universal property as it is about every effective open set.

For instance, we mentioned that the subclass LCE $\subsetneq \Delta_{2}^{0}$ is meager in $\Delta_{2}^{0}$, i.e., typical $\Delta_{2}^{0}$ sets are not left-c.e. Actually, no 1-generic set is left-c.e. Indeed, 
if $x \in[0,1]$ is left-c.e. then the interval $[0, x)$ is an effective open set that is dense along $x$, i.e., contains $x$ in its boundary.

In the same way as Baire Cateory provides a simple way of proving existence results without giving explicit constructions, 1-genericity can be easily used to

prove existence of $\Delta_{2}^{0}$-sets with prescribed properties, and is an alternative to explicit constructions.

A famous example is Kleene-Post's theorem, on the way to the solution to Post's problem:

Theorem 3.1 (Kleene and Post [10]). There exist two Turing-incomparable $\Delta_{2}^{0}$ sets, i.e. two $\Delta_{2}^{0}$-sets that are not computable relative to each other.

Actually for a typical $\Delta_{2}^{0}$-set $A$, its two halves $A_{0}=\{n \in \mathbb{N}: 2 n \in A\}$ and $A_{1}=\{n \in \mathbb{N}: 2 n+1 \in A\}$ are Turing-incomparable $\Delta_{2}^{0}$-sets. Moreover,

Theorem 3.2 (Jockush, [8]). Every 1-generic set has Turing-incomparable halves.

Proof. This can be proved very easily: given a Turing machine $M$, let $\mathcal{U}=\{A$ : $\exists n, M^{A_{0}}(n)=0$ but $\left.n \in A_{1}\right\}$. $\mathcal{U}$ is an effective open class and if $A_{1}$ is Turing reducible to $A_{0}$ via $M$ then $A$ belongs to the boundary of $U$ : adding an arbitrary large element to $A_{1}$ makes $A$ fall in $\mathcal{U}$ (possible when $A_{1}$ is co-infinite). Hence if $A$ is 1-generic, $A$ does not belong to the boundary of $\mathcal{U}$ and as $A_{1}$ is easily co-infinite, $M$ does not compute $A_{1}$ relative to $A_{0}$.

Hence instead of constructing a $\Delta_{2}^{0}$-set with the specific property, one simply has to check that the property is co-meager in $\Delta_{2}^{0}$, or even that it is captured by 1-genericity. The relativized computable Baire Category theorem gives the existence result for free.

\section{Enumerable objects}

As we saw, the case of computable sets is a straightforward effectivization of the ordinary, non-effective setting, from which the case of $\Delta_{2}^{0}$-sets is a straightforward relativization.

We now turn our attention to intermediate classes of objects. What is a small class inside the class $C E$ of c.e. subsets of $\mathbb{N}$ ? What is a typical c.e. set? The same questions can be asked for left-c.e. sets, $\Pi_{1}^{0}$-classes, etc.

\section{$4.1 \quad$ C.e. sets}

First observe that as $\mathrm{C} \subseteq \mathrm{CE}$, we can declare every class that is meager in $\mathrm{C}$ to be meager in CE. Axiom 1. is satisfied, i.e., every singleton is meager in CE: (i) if $A \subseteq \mathbb{N}$ is finite then it is computable so as before $\{A\}$ is meager as its complement is a dense effective open set, (ii) if $A$ is infinite then the class $\mathcal{U}=$ $\{B \subseteq \mathbb{N}: A \nsubseteq B\}$ is a dense effective open set that does not contain $A$. However 
Axiom 4. is not satisfied as $\mathrm{C}$ is meager in $\mathrm{CE}$, so we have to add new meager classes in CE.

The idea is to weaken the definition of 1-genericity. Let us recall that a set $A \subseteq \mathbb{N}$ is 1-generic if it does not belong to the boundary of any effective open class $\mathcal{U} \subseteq 2^{\mathbb{N}} ; A$ belongs to the boundary of $\mathcal{U}$, or $\mathcal{U}$ is dense along $A$, means that $\mathcal{U}$ contains sets arbitrarily close to $A$, i.e., in every cylinder $\left[A \uparrow_{n}\right]$.

Definition 4.1. We say that $A$ belongs to the down-closure of $\mathcal{U}$, or that $\mathcal{U}$ is dense above $A$, if $\mathcal{U}$ contains sets $B \supseteq A$ arbitrarily close to $A$, i.e., in every cylinder $\left[A \uparrow_{n}\right]$. We say that $A$ belongs to the down-boundary of $\mathcal{U}$ if $A$ belongs to the down-closure of $\mathcal{U}$ but not to $\mathrm{U}$.

Observe that the down-boundary of $\mathcal{U}$ is contained in the boundary of $\mathcal{U}$, so if $\mathcal{U}$ is an effective open set then its down-boundary is meager in $\Delta_{2}^{0}$. We declare that its down-boundary is already meager in CE. It gives a notion of genericity in the class CE.

Definition 4.2. A set $A$ is generic from above if it belongs to every effective open class that is dense above $A$. In other words, $A$ is generic from above if it does not belong to the down-boundary of any effective open class.

This notion is equivalent to the notion of $p$-genericity introduced by Ingrassia [7]. We then prove a Baire Category theorem in CE.

Theorem 4.3 (Baire Category in CE $[7,5]$ ). Let $\mathcal{U}_{n} \subseteq 2^{\mathbb{N}}$ be dense uniformly effective open classes. The class of c.e. sets in $\bigcap_{n} \mathcal{U}_{n}$ that are generic from above is dense in $2^{\mathbb{N}}$.

As a result, the class CE is not meager in itself and Axiom 2. is satisfied. Moreover Axiom 4. is satisfied, i.e., the subclass $\mathrm{C}$ is meager in CE: (i) the class of co-finite sets is already meager in $\mathrm{C}$ as it can be effectively listed, (ii) if $A \subseteq \mathbb{N}$ is co-infinite and computable then it is not generic from above, as the effective open set $2^{\mathbb{N}} \backslash\{A\}$ is dense above $A$.

Genericity from above is a weakening of 1-genericity, that is sometimes sufficient for our purpose. For instance the simple argument showing that the two halves of a 1-generic set are Turing-incomparable (Theorem 3.2) immediately applies to co-infinite sets that are generic from above. As a result, Theorem 4.3 implies the solution to Post problem invented by Friedberg and Muchnik.

Theorem 4.4 (Friedberg-Muchnik $[\mathbf{1 3}, 4]$ ). There exist two Turing-incomparable c.e. sets.

Additionally, we can say that having Turing-incomparable halves is a typical property of c.e. sets, and every c.e. set that is generic from above has this property.

Obverse that it does not give an alternative proof of Friedberg-Muchnik's theorem, as Theorem 4.3 is showed using the priority method with finite injury, the method invented by Friedberg and Muchnik to prove their result. However, 
many constructions using a simple form of the priority method are captured by the Baire Category theorem in CE. For instance, every co-finite set $A$ that is generic from above is not autoreducible: there is no Turing functional that for each $n \in \mathbb{N}$, decides $n \in A$ given $A \backslash\{n\}$ as oracle.. Not all finite injury arguments are captured by Theorem 4.3: for instance Ingrassia [7] proved the existence of a $p$-generic (i.e., co-infinite and generic from above) c.e. set that is Turing equivalent to the halting set, hence is not a solution to Post's problem.

\subsection{Left-c.e. reals}

We can adapt the previous definitions in a straightforward way, replacing the inclusion ordering over $2^{\mathbb{N}}$ by the lexicographic ordering. Identifying subsets of $\mathbb{N}$ with real numbers in $[0,1]$, we get the natural ordering of real numbers.

Definition 4.5. We say that $x \in[0,1]$ belongs to the left-closure of $\mathcal{U} \subseteq[0,1]$, or that $\mathcal{U}$ is dense on the right of $x$, if $\mathcal{U}$ contains reals $y \geq x$ arbitrarily close to $x$, i.e., in every interval $[x, x+\epsilon)$. We say that $x$ belongs to the left-boundary of $\mathcal{U}$ if $x$ belongs to the left-closure of $\mathcal{U}$ but not to $\mathrm{U}$.

For instance, if $0<a<1$ then the left-boundary of $(a, 1]$ is $\{a\}$ while the leftboundary of $[0, a)$ is empty. In particular, while every left-c.e. real $a>0$ belongs to the boundary of the effective open set $[0, a)$, preventing it from being 1-generic, it does not belong to its left-boundary.

We then declare the left-boundary of an effective open class to be meager in LCE, and we get a notion of genericity.

Definition 4.6. A real $x$ is generic from the right if it belongs to every effective open class that is dense on the right of $x$. In other words, $x$ is generic from the right if $x$ does not belong to the left-boundary of any effective open class.

Again we have a Baire Category theorem in LCE.

Theorem 4.7 (Baire Category in LCE). The class of left-c.e. reals that are generic from the right is non-empty and dense.

Observe that we do not need to intersect with an effective family of dense open sets as in Theorem 4.3, as every dense set is also dense on the right of every $x<1$. It means that every real that is generic from the right is weakly1-generic, i.e., belongs to every dense effective open set. The construction of a weakly-1-generic left-c.e. real presented in [14] actually builds a real that is generic on the right, hence proves Theorem 4.7.

Again the theorem implies that Axiom 2. is satisfied, i.e., that LCE is not meager in itself. Axiom 4. is satisfied as CE is meager in LCE: we know from the previous section that every c.e. set it outside some dense effective open set.

However this time Axiom 1. is not satisfied! By definition a left-c.e. real that is generic on the right does not belong to the left-boundary of any effective open class. We need to add more nowhere dense classes. However we were unable to identify a natural way of doing it. 


\subsection{Other classes of objects}

In [5] we introduce a way of defining a notion of genericity for more general classes of enumerable objects and prove a Baire Category theorem for such classes. For instance, one can define what is a generic $\Pi_{1}^{0}$ subset of $2^{\mathbb{N}}$. We apply this method to obtain a result in computable analysis related to the non-computability of the ergodic decomposition theorem. In [6] we give other applications of this method showing that many complicated constructions in recursion theory can be more easily obtained by choosing the suitable topology on the space of objects, and using the corresponding Baire Category theorem on that space.

\section{Conclusion}

For many classes of constructible objects it is possible to define a notion of meager subclass and a corresponding notion of genericity. A typical element of the class is then generic and automatically satisfies many interesting properties. The relevance of these notions can be measured in two ways that oppose to each other:

- A Baire Category theorem should hold, i.e., the class should not be meager in itself. Said differently, the notion of genericity should not be too strong.

- The notion of genericity should be strong enough to capture many useful interesting properties.

When these conditions are satisfied, existence results become easy to derive. In this note, the Baire Category theorem for classes of enumerable objects is proved using the simplest form of priority method with finite injury. A future direction would be to define weaker notions of meager subclass, or stronger notions of genericity, capturing more advanced methods from recursion theory such as the priority method with infinite injury.

\section{References}

1. Vasco Brattka, Matthew Hendtlass, and Alexander P. Kreuzer. On the uniform computational content of the Baire category theorem. CoRR, abs/1510.01913, 2015.

2. Douglas Bridges, Hajime Ishihara, and Luminița Vîţ̆ă. A new constructive version of Baire's theorem. Hokkaido Math. J., 35(1):107-118, 022006.

3. Douglas K. Brown and Stephen G. Simpson. The Baire category theorem in weak subsystems of second-order arithmetic. J. Symbolic Logic, 58(2):557-578, 061993.

4. Richard M. Friedberg. Two recursively enumerable sets of incomparable degrees of unsolvability (solution of Post's problem, 1944). Proceedings of the National Academy of Sciences of the United States of America, 43(2):236-238, 1957.

5. Mathieu Hoyrup. Irreversible computable functions. In Ernst W. Mayr and Natacha Portier, editors, 31st International Symposium on Theoretical Aspects of Computer Science (STACS 2014), STACS 2014, March 5-8, 2014, Lyon, France, volume 25 of LIPIcs, pages 362-373. Schloss Dagstuhl - Leibniz-Zentrum fuer Informatik, 2014. 
6. Mathieu Hoyrup. Genericity of weakly computable objects. To appear in Theory of Computing Systems, 2016.

7. M.A. Ingrassia. P-genericity for Recursively Enumerable Sets. PhD thesis, University of Illinois at Urbana-Champaign, 1981.

8. Carl G. Jockush. Simple proofs of some theorems on high degrees. Canad. J. Math., 29:1072-1080, 1977.

9. Sara Hawtrey Jones. Applications of the Baire category theorem. Real Anal. Exchange, 23(2):363-394, 1999.

10. S. C. Kleene and Emil L. Post. The upper semi-lattice of degrees of recursive unsolvability. Annals of Mathematics, 59(3):379-407, 1954.

11. Jack H. Lutz. Category and measure in complexity classes. SIAM J. Comput., 19(6):1100-1131, 1990.

12. Jack H. Lutz. Effective fractal dimensions. Mathematical Logic Quarterly, 51(1):62$72,2005$.

13. A. A. Muchnik. On the unsolvability of the problem of reducibility in the theory of algorithms. Dokl. Akad. Nauk SSSR, 108:194-197, 1956.

14. André Nies. Computability and randomness. Oxford logic guides. Oxford University Press, 2009. 